\title{
Polarization-dependent Landau level crossing in a two-dimensional electron system in a MgZnO/ZnO heterostructure
}

\author{
D. Maryenko, ${ }^{1,}{ }^{*}$ J. Falson, ${ }^{2}$ Y. Kozuka, ${ }^{2}$ A. Tsukazaki, ${ }^{3}$ and M. Kawasaki ${ }^{1,2}$ \\ ${ }^{1}$ RIKEN Center for Emergent Matter Science (CEMS), Wako 351-0198, Japan \\ ${ }^{2}$ Department of Applied Physics and Quantum-Phase Electronics Center (QPEC), The University of Tokyo, Tokyo 113-8656, Japan \\ ${ }^{3}$ Institute for Materials Research, Tohoku University, Sendai 980-8577, Japan
}

(Received 21 October 2014; revised manuscript received 3 December 2014; published 18 December 2014)

\begin{abstract}
We report electrical transport measurements in a tilted magnetic field on a high-mobility two-dimensional electron system (2DES) confined at the $\mathrm{MgZnO} / \mathrm{ZnO}$ heterointerface. The observation of multiple crossing events of spin-resolved Landau levels (LLs) enables the mapping of the sequence of electronic states. We further measure the renormalization of electron spin susceptibility at zero field and the susceptibility dependence on the electron spin polarization. The latter manifests the deviation from the Pauli spin susceptibility. As a result, the crossing of spin-resolved LLs shifts to smaller tilt angles and the first Landau level coincidence event is absent even when the magnetic field has only a component perpendicular to the 2DES plane.
\end{abstract}

DOI: 10.1103/PhysRevB.90.245303

PACS number(s): 77.55.hf, 71.18.+y, 71.70.-d, 73.43.-f

\section{INTRODUCTION}

A magnetic field applied to a two-dimensional electron system (2DES) opens several energy gaps in the electronic spectrum. In a single-particle picture, two fundamental energy scales dominate there. One is the Zeeman gap $E_{\mathrm{z}}=$ $g^{*} \mu_{B} B_{\text {total }}$, which separates the spin states in the total magnetic field $B_{\text {total }}$. Here $g^{*}$ is the Landé $g$ factor and $\mu_{B}$ is the Bohr magneton. The other is the cyclotron gap $E_{\text {cyc }}$ and reflects the quantization of electron orbital motion by the formation of Landau levels (LLs) with energy $\hbar \omega_{c}(N+1 / 2)$, where $N$ is the LL index and $\omega_{c}=e B_{\perp} /\left(m^{*} m_{0}\right)$ is the cyclotron frequency given by the field component normal to the 2DES plane, $B_{\perp}=B_{\text {total }} \cos (\theta)$. Here, $\theta$ is the angle between the direction of $B_{\text {total }}$ and the normal to the sample, $\hbar$ is the reduced Planck constant, $e$ is the elementary charge, and $m^{*}$ is the electron effective mass in units of the free-electron mass $m_{0}$. The ratio of the number of electrons to the LL degeneracy, given by the amount of magnetic flux threading the sample, $e B_{\perp} / h$, defines the filling factor $v$ of this electron energy ladder and indicates how many levels are occupied.

The two energy scales can be tuned relative to each other by tilting the sample in $B_{\text {total }}$; the electron states at different LLs and with opposite spin orientations can eventually be brought into coincidence. This method has been applied for various $2 \mathrm{DESs}$ to evaluate their electron spin susceptibility $\chi$, a fundamental property in condensed matter physics that describes the response of spin polarization $P$ to changes of the applied magnetic field $B_{\text {total }}$ [1-10]. It relies, however, on the Pauli spin susceptibility with linear dependence:

$$
P=\frac{\chi}{n} B_{\text {total }}=\frac{e}{2 h} \frac{g^{*} m^{*}}{n} B_{\text {total }},
$$

where $n$ is the electron density. Such linearity is not a given in a strongly interacting 2DES. Rather, $\chi$ depends on $P$ and therefore one can question the reliability of the coincidence method for evaluating $\chi$ [11]. A high-electronmobility $\mathrm{MgZnO} / \mathrm{ZnO}$ heterostructure is particularly suitable

\footnotetext{
*maryenko@ riken.jp
}

for probing this concept $[12,13]$. First, the cyclotron and Zeeman gaps are comparable in size, i.e., $E_{\text {cyc }} / E_{\mathrm{z}} \approx 3.3$ at $\theta=0$, when both the band electron mass $\left(m^{*}=0.3\right)$ and the Landé $g$ factor $\left(g^{*}=2\right)$ are considered. Therefore, level coincidences may be achieved at moderate $\theta$ and consequently multiple coincidence positions, signifying a large polarization range, may be observed. This too may allow the mapping of these transitions and the polarization. Second, the 2DES at $\mathrm{MgZnO} / \mathrm{ZnO}$ heterointerfaces is a strongly interacting system with a reported enhancement of both the electron mass and the Landé $g$ factor [14-16]. Thus, this system ought to show a pronounced dependence of $\chi$ on $P$.

In this paper, we describe magnetotransport experiments on a $2 \mathrm{DES}$ confined at the $\mathrm{MgZnO} / \mathrm{ZnO}$ heterointerface by rotating the sample in the magnetic field in small steps (ca. $0.25^{\circ}$ ) from $\theta=0$ up to $\theta$ approaching $90^{\circ}$ and thus carefully examine Landau level crossing events.

\section{EXPERIMENTAL DETAILS}

The sample has a van der Pauw geometry with eight indium Ohmic contacts soldered at the corners and at the side centers of the sample as shown in Fig. 1(c). The mobility of the heterostructure is about $300000 \mathrm{~cm}^{2} / \mathrm{V} \mathrm{s}$ and its electron density $n=2.05 \times 10^{11} \mathrm{~cm}^{-2}$ [17]. The measurements are done in a ${ }^{3} \mathrm{He}$ refrigerator at $T \approx 500 \mathrm{mK}$. The sample is mounted on a single-axis rotation stage, which allows variation of $\theta$ in situ. The zero tilt angle is adjusted by rotating the sample in $0.5 \mathrm{~T}$ magnetic field until the maximum value of the Hall voltage is achieved. For a fixed $\theta$, the longitudinal resistance $R_{x x}$ and the Hall resistance $R_{x y}$ are recorded as functions of $B_{\text {total }}$. The measurements are done using a standard lock-in measurement technique at $11.3 \mathrm{~Hz}$ and an excitation current of $100 \mathrm{nA}$. The tilt angle is deduced by adjusting the slope of the Hall voltage and by adjusting the pronounced $R_{x x}$ minima.

\section{EXPERIMENTAL RESULTS AND DISCUSSIONS}

Figure 1(a) is a color rendition plot of $R_{x x}$ as a function of $B_{\perp}$ and $1 / \cos (\theta)$ with red (blue) areas representing high 

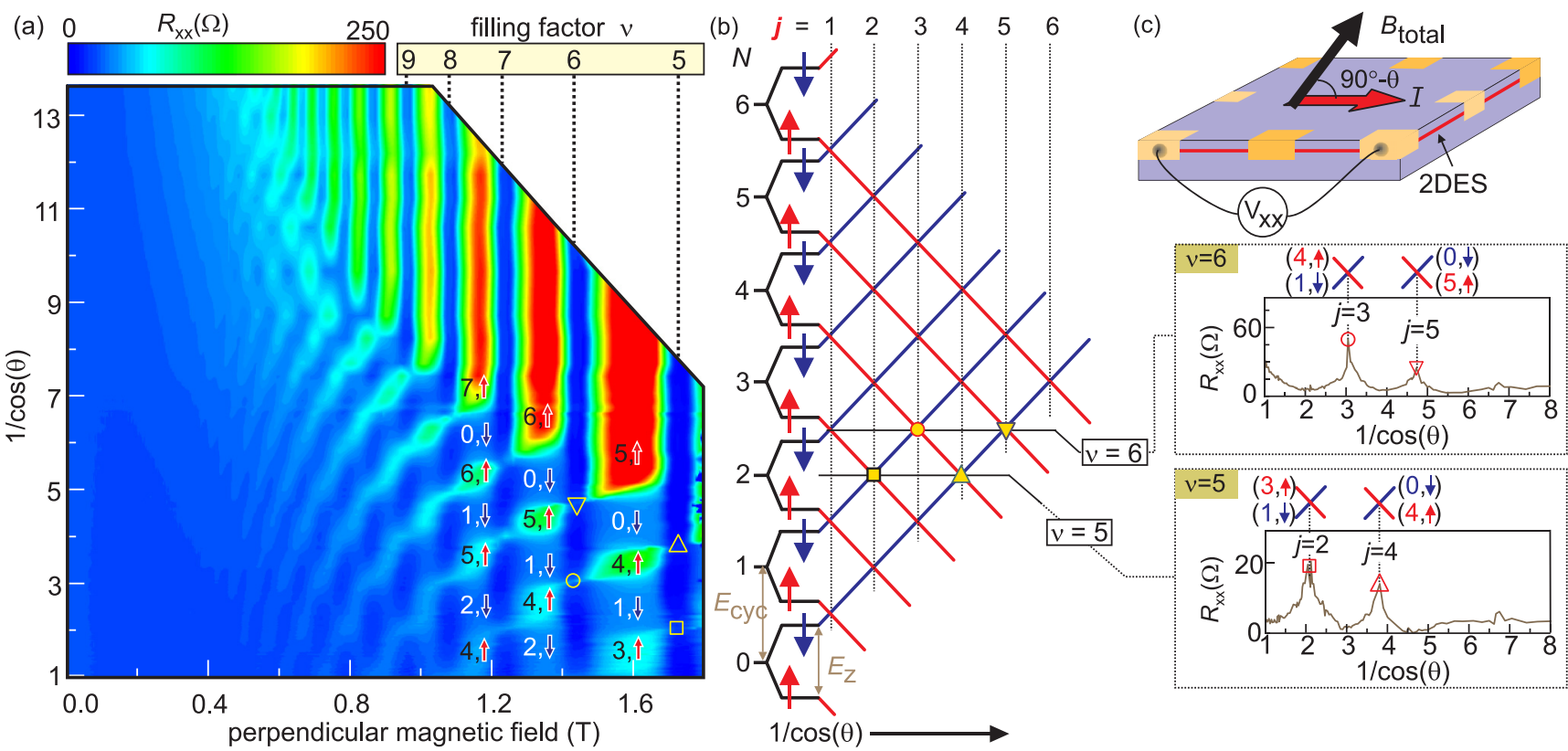

FIG. 1. (Color online) (a) Color rendition plot of $R_{x x}$ as a function of $B_{\perp}$ and $1 / \cos (\theta)$. Indicated are the electron states $(N$, $\uparrow$ or $\downarrow)$ at the chemical potential. (b) Scheme of LL crossings in a magnetic field assuming a fixed $B_{\perp}$ (left panel). $j$ indicates the index of LL crossings. $R_{x x}$ traces are shown for $v=5$ and 6 as functions of $1 / \cos (\theta)$. (c) Schematic of the sample. The sample is tilted in $B_{\text {total }}$ so that the direction of the in-plane field is collinear with the current direction.

(low) $R_{x x}$ values. First, we affirm that the observed resistance changes are associated with the crossing of spin-resolved LLs. We start by drawing in Fig. 1(b) the fan diagram of spin-resolved LLs for a fixed $B_{\perp}$ in an increasing $B_{\text {total }}$ represented by the $1 / \cos (\theta)$ axis and assume for the moment a constant $\chi$. For a given $B_{\perp}$, the energy of spin-up $\uparrow$ (spin-down $\downarrow)$ electron states decreases (increases) in an increasing $B_{\text {total }}$ as indicated by red (blue) lines in Fig. 1(b). For the discussion below, we introduce the notation $(N, \uparrow$ or $\downarrow)$ to characterize the electron state. Such a diagram visualizes the possible LL crossings, which show up in the experiment as an $R_{x x}$ increase at the respective $\nu$. Figure 1(b) exemplifies $R_{x x}$ traces for $v=5$ and 6 as functions of $1 / \cos (\theta)$, extracted from Fig. 1(a). Horizontal lines indicate the positions of the corresponding chemical potentials. When the chemical potential lies within the spectrum's gap, $R_{x x}$ tends toward zero. Whenever two levels cross at the chemical potential, the resistance rises. Accordingly the traces exhibit characteristic sharp peaks and thus signal both the LL crossing and the change of the overall 2DES polarization. For $v=5$, the diagram implies two crossing events; one is the crossing of $(N=1, \downarrow)$ with $(N=3, \uparrow)$ and the other is $(N=0, \downarrow)$ crossing with $(N=$ $4, \uparrow)$. Experimentally, two resistance peaks are observed and an index, respectively $j=2$ or $j=4$, can be assigned to each of them. Interestingly, the $R_{x x}$ trace for $v=6$ shows also two coincidence events with $j=3$ and 5, although the fan diagram suggests that three such events should occur. We note that this experimental trace shows a tail of the resistance peak, which can be associated with $j=1$ at around $1 / \cos (\theta) \approx 1$. Such behavior implies $\chi$ enhancement by so much that the first coincidence has already occurred even at $\theta=0$, consistent with previous reports $[14,15]$. This analysis identifies also the electronic states at the chemical potential in each region of
Fig. 1(a) and thus establishes the map of electronic states in the magnetic field.

The level crossing takes place whenever $j E_{\text {cyc }}=E_{\mathrm{z}}$; the integer $j$ characterizes the difference between LL orbital momenta $N_{\uparrow}-N_{\downarrow}$. Taking into account that $B_{\perp}=B_{\text {total }} \cos (\theta)$, the coincidence condition is rewritten

$$
\frac{g^{*} m^{*}}{2}=j \cos (\theta)
$$

which is the expression frequently used to estimate $\chi$. Accordingly, Fig. 2 plots $1 / \cos (\theta)$, at which the level crossing happens, versus $j$ for several $v$ 's. For $j \leqslant 3$ all points fall onto a straight line, which passes through the origin and has a slope

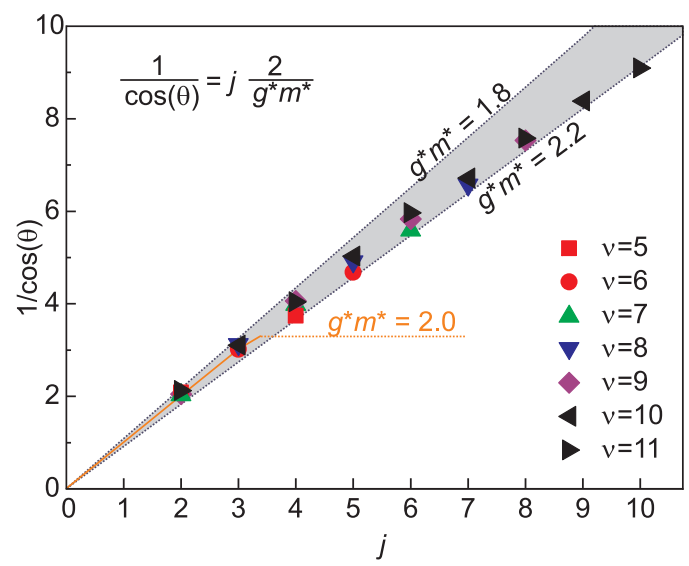

FIG. 2. (Color online) Estimation of spin susceptibility at several $v$ 's. The inset equation describes the linear dependence with $g^{*} m^{*}=$ 2.0 for $j \leqslant 3$. The gray shaded area indicates a range of $g^{*} m^{*}$ of 1.8 (upper edge) to 2.2 (lower edge). 


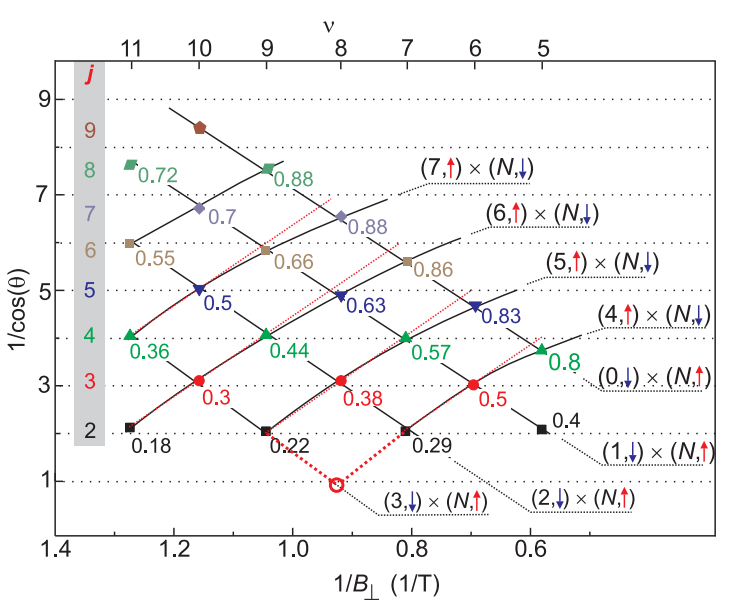

FIG. 3. (Color online) Positions of LL crossings (closed symbols) at integer $v$ and angles $\theta$ extracted from Fig. 1(a). The spin polarization value $P$ is given at each crossing point. The solid lines are guides to the eye. A dashed red line emphasizes a systematic shift of coincidence events to smaller $1 / \cos (\theta)$. The open circle is one of the $j=1$ crossings obtained by extrapolating experimental data points. It lies at $1 / \cos (\theta)<1$ and emphasizes that the first crossing event has occurred in a purely perpendicular magnetic field.

corresponding to $g^{*} m^{*}=2.0$. This magnitude is consistent with our previous measurements which focused on $j \leqslant 3$ and it was thought to represent the spin susceptibility at zero field $[14,15]$. However, for larger $\theta$, explored in this work, the points deviate from this linear dependence when $j>3$ and systematically shift to a smaller $1 / \cos (\theta)$ for larger $j$ 's. This reveals some nonlinearity in the 2DES and a departure from the level crossing diagram in Fig. 1(b). This in turn necessitates a reconsideration of the spin susceptibility at zero field.

To study the level crossing in more detail, Fig. 3 summarizes the crossing events that are unambiguously identified from gap closing at integer $v$. Both the color and shape of the symbols group the coincidence events with the same $j$. The solid curves are guides to the eye and connect particular crossing events. The lines running from top left to bottom right represent the crossings of $(N, \downarrow)$ with fixed $N$ with $\left(N^{\prime}, \uparrow\right)$. The lines running from bottom left to top right indicate the events when $(N, \uparrow)$ with $N=4,5,6$, etc., cross $\left(N^{\prime}, \downarrow\right)$ with an arbitrary $N^{\prime}$. This mapping shows the absence of $j=1$ coincidence; a red open circle at $1 / \cos (\theta)<1$ denotes the position of the hypothetical $j=1$ crossing by extrapolating the experimental results. More importantly, we find that the crossing events with the same $j$ occur at a smaller $\theta$, i.e., a smaller $1 / \cos (\theta)$, for smaller $v$ 's. It appears to correlate well with the electron spin polarization estimated as $P=\left(n_{\uparrow}-n_{\downarrow}\right) /\left(n_{\uparrow}+n_{\downarrow}\right)=j / \nu$. The $P$ values are depicted in Fig. 3 next to each crossing point. Hence, the larger is $P$, the smaller is $\theta$ at which the crossing takes place. Effectively, this corresponds to the increase of $\chi$ with increasing $P$.

We scrutinize the role of $P$ by plotting it as a function of $B_{\text {total }}$ in Fig. 4(a). This plot covers a wide polarization range, which otherwise is achieved only in InSb-based 2DESs [18], and shows a nonlinear dependence of $P$ on $B_{\text {total }}$. This nonlinearity is captured by using Eq. (1) and assuming a phenomenological relation $\chi=\chi_{0}+\Delta \chi P$, where $\chi_{0}$ is the
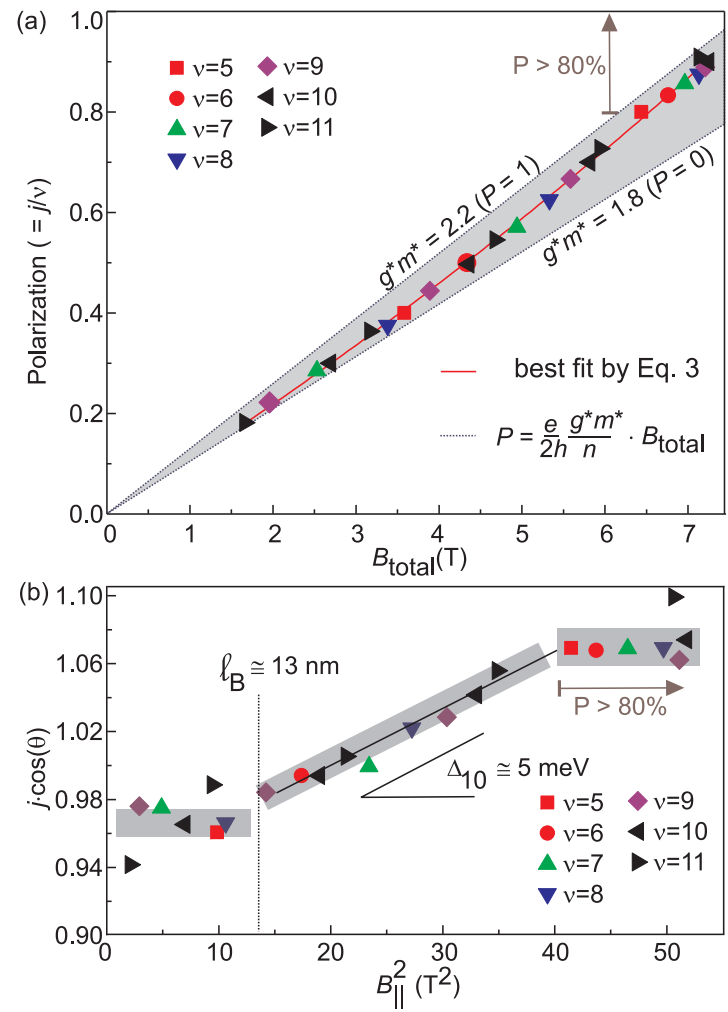

FIG. 4. (Color online) (a) Spin polarization (estimated from Fig. 3) vs total magnetic field. The nonlinearity indicates a polarization-dependent spin susceptibility. The experimental points are bounded by (dashed) lines of constant $\chi$ corresponding to $P=0$ and $P=1$, respectively. (b) Analysis of in-plane field effect on LL crossing yields the subband separation $\Delta_{10}=5 \mathrm{meV}$ and the electron wave function extension $13 \mathrm{~nm}$.

spin susceptibility at $B_{\text {total }}=0$ and $\Delta \chi$ describes the rate of $\chi$ enhancement $[3,18,19]$. We obtain then

$$
P=\frac{\chi_{0} B_{\text {total }}}{n-\Delta \chi B_{\text {total }}}
$$

This dependency describes well the $P-B_{\text {total }}$ nonlinearity in Fig. 4(a) with the best-fit parameters (red line) $\chi_{0}=$ $1.77 \pm 0.02$ and $\Delta \chi=0.38 \pm 0.03$, both in units of $e / 2 h$. Accordingly, the experimental points fall into the area bounded by two lines representing a constant 2DES spin susceptibility with $\chi_{0}$, i.e., $P=0$, and with $\chi_{0}+\Delta \chi$, i.e., $P=1$. These lines are also shown in Fig. 2, where they too represent the bounds for the experimental data and demonstrate the error caused by using coincidence techniques to evaluate $\chi$.

Table I compares the spin susceptibilites in $\mathrm{ZnO}-$, AlAs-, and GaAs-based high-mobility 2DESs with the same WignerSeitz interaction parameter $r_{s} \approx 8$. We note, however, that the result for GaAs-based [3] and AlAs-based [20] 2DESs were obtained using a conventional Landau level coincidence technique. The estimated value $\chi_{0}=1.77 \mathrm{in} \mathrm{ZnO}$ is a threefold enhancement compared to the band value $\chi_{\text {band }}=0.6$ in $\mathrm{ZnO}$ and thus is comparable with $\chi_{0}$ enhancement in AlAs. We point out that the conventional coincidence method for $\mathrm{ZnO}$ heterostructures yields a zero-field value $\chi\left(=2.0 \frac{e}{2 h}\right)$ overestimated by $12 \%$. Since $\chi$ has a stronger dependence on 
TABLE I. Comparison of spin susceptibility(in units of $e / 2 h$ ) for $\mathrm{ZnO}-$, AlAs-, and GaAs-based 2DESs with charge carrier density corresponding to the Wigner-Seitz radius $r_{s}=8$. The data for GaAs and AlAs are extracted from Refs. [3,20], respectively. Here, $\chi_{\text {band }}$ is the band susceptibility and $P$ is the net spin polarization.

\begin{tabular}{lllcc}
\hline \hline 2DES & $\chi_{\text {band }}$ & $\chi=\chi_{0}+\Delta \chi P$ & $\Delta \chi / \chi_{0}$ & $\chi / \chi_{\text {band }}$ \\
\hline ZnO & 0.6 & $1.77+0.38 \mathrm{P}$ & $21 \%$ & $2.95+0.63 \mathrm{P}$ \\
$\mathrm{AlAs}$ & 0.92 & $2.76+0.36 \mathrm{P}$ & $13 \%$ & $3+0.4 \mathrm{P}$ \\
GaAs & 0.029 & $0.10+0.075 \mathrm{P}$ & $75 \%$ & $3.6+2.57 \mathrm{P}$ \\
\hline
\end{tabular}

$P$ in GaAs, the $\chi_{0}$ evaluation can be more strongly affected when the conventional coincidence method is employed.

\section{EFFECT OF THE IN-PLANE FIELD}

We discuss now whether the $\chi$ enhancement in $\mathrm{ZnO}$ is the result of the in-plane magnetic field coupling to the electron orbital motion. Such a coupling is known to mix the subbands of the confinement potential and to enhance the electron effective mass, which in turn enhances $\chi$ [21-23]. When only the coupling between the ground and the first excited states is considered, the mass increases by a factor $1+\frac{1}{2}\left(\frac{\hbar \omega_{\|}}{\Delta_{10}}\right)^{2}$, where $\omega_{\|}=e B_{\|} /\left(m^{*} m_{0}\right)$ with $B_{\|}$being the in-plane field component and $\Delta_{10}$ the subband splitting. Then the coincidence condition attains an in-plane field dependence

$$
j \cos (\theta)=\frac{1}{2} g^{*} m^{*} \approx 1+\frac{1}{2}\left(\frac{\hbar e / m^{*} m_{0}}{\Delta_{10}} B_{\|}\right)^{2} .
$$

This model does not include the electron correlation effects and thus provides an upper bound on the in-plane field effect. Figure 4(b) analyzes this model and plots $j \cos (\theta)$ versus $B_{\|}^{2}$. For small $B_{\|}$, no noticeable field dependence is observed, indicating no significant effect of the orbital coupling. However, starting at $B_{\|}^{2} \approx 15 \mathrm{~T}^{2}$ a linear dependence persists up to $B_{\|}^{2}=40 \mathrm{~T}^{2}$. In this regime the effect of the in-plane field can account for $8 \%$ of the mass enhancement. Thereafter no field dependence is observed again, suggesting a saturation of the orbital coupling effect and no further electron mass enhancement. Therefore, the $\chi$ dependence discussed above originates primarily from electron correlation effects, with some (8\%) contribution from the electron mass enhancement caused by in-plane field coupling. According to the theory presented in Refs. [22-25], the mass saturation is not expected. Rather the mass should grow linearly with $B_{\|}^{2}$. However, it is also known that the polarization of the electron system suppresses the effective mass [26-28]. Therefore, this mechanism can counteract the further increase of the electron mass. This is likely to happen in our system, since the saturation effect in our system occurs for $P>80 \%$.

For the sake of analysis, we exploit the constructed model of the in-plane field effect and estimate the parameters of the electron confinement potential, for which data obtained through transport experiments in $\mathrm{MgZnO} / \mathrm{ZnO}$ heterostructures appear to be lacking. First, the slope of the linear dependence yields a lower bound for the subband separation of the confinement potential $\Delta_{10} \cong 5 \mathrm{meV}$. It is larger than the Fermi energy of this $2 \mathrm{DES}, 1.6 \mathrm{meV}$, and confirms the single-band population. Second, the coupling of $B_{\|}$to the orbital motion is effective if the radius of the electron orbit, characterized by the magnetic length $l_{B}$, becomes comparable with the extension of the subband wave function. Therefore, we estimate its extension of $13 \mathrm{~nm}$ from the field at which the linear dependence sets in.

\section{CONCLUSION}

In conclusion, electrical transport experiments in a tilted magnetic field mapped out the sequence of electronic states in a $\mathrm{MgZnO} / \mathrm{ZnO}$ heterostructure. Due to the observation of multiple coincidence events, we evaluated a nonlinear dependence of the net spin polarization on the total magnetic field. For a strongly interacting electron system this is in line with the theory of Ref. [11]. We further measured the polarization-dependent spin susceptibility $\chi=1.77+0.38 P$ for $r_{s} \approx 8$ and thus revealed a deviation from the Pauli spin susceptibility. This observation along with the spin susceptibility renormalization at $B_{\text {total }}=0$ supports the theory that $\mathrm{ZnO}$-based 2DESs are interacting Fermi liquids. Our result demonstrates that the position of LL crossings depends on the spin polarization in a strongly interacting system. As a consequence, the conventional coincidence method, yielding $\chi=2.0 \frac{e}{2 h}$, overestimates the zero-field spin susceptibility.

\section{ACKNOWLEDGMENTS}

We would like to thank J. H. Smet, D. Zhang, B. Friess, and L. Tiemann for fruitful discussions. This work was partly supported by the Grant-in-Aid for Scientific Research (S) No. 24226002 from MEXT, Japan, and the "Funding Program for World-Leading Innovative R\&D on Science and Technology (FIRST)" Program from the Japan Society for the Promotion of Science (JSPS) initiated by the Council for Science and Technology Policy.
[1] F. F. Fang and P. J. Stiles, Phys. Rev. 174, 823 (1968).

[2] R. J. Nicholas, R. J. Haug, K. v. Klitzing, and G. Weimann, Phys. Rev. B 37, 1294 (1988).

[3] J. Zhu, H. L. Stormer, L. N. Pfeiffer, K. W. Baldwin, and K. W. West, Phys. Rev. Lett. 90, 056805 (2003).

[4] K. Vakili, Y. P. Shkolnikov, E. Tutuc, E. P. De Poortere, and M. Shayegan, Phys. Rev. Lett. 92, 226401 (2004).

[5] V. M. Pudalov, M. E. Gershenson, H. Kojima, N. Butch, E. M. Dizhur, G. Brunthaler, A. Prinz, and G. Bauer, Phys. Rev. Lett. 88, 196404 (2002).
[6] A. A. Shashkin, S. V. Kravchenko, V. T. Dolgopolov, and T. M. Klapwijk, Phys. Rev. Lett. 87, 086801 (2001).

[7] A. A. Shashkin, M. Rahimi, S. Anissimova, S. V. Kravchenko, V. T. Dolgopolov, and T. M. Klapwijk, Phys. Rev. Lett. 91, 046403 (2003).

[8] E. Tutuc, S. Melinte, and M. Shayegan, Phys. Rev. Lett. 88, 036805 (2002).

[9] S. A. Vitkalov, H. Zheng, K. M. Mertes, M. P. Sarachik, and T. M. Klapwijk, Phys. Rev. Lett. 87, 086401 (2001). 
[10] S. Brosig, K. Ensslin, A. G. Jansen, C. Nguyen, B. Brar, M. Thomas, and H. Kroemer, Phys. Rev. B 61, 13045 (2000).

[11] Y. Zhang and S. Das Sarma, Phys. Rev. Lett. 96, 196602 (2006).

[12] A. Tsukazaki et al., Science 315, 1388 (2007).

[13] A. Tsukazaki et al. , Nat. Mater. 9, 889 (2010).

[14] A. Tsukazaki, A. Ohtomo, M. Kawasaki, S. Akasaka, H. Yuji, K. Tamura, K. Nakahara, T. Tanabe, A. Kamisawa, T. Gokmen, J. Shabani, and M. Shayegan, Phys. Rev. B 78, 233308 (2008).

[15] Y. Kozuka, A. Tsukazaki, D. Maryenko, J. Falson, C. Bell, M. Kim, Y. Hikita, H. Y. Hwang, and M. Kawasaki, Phys. Rev. B 85, 075302 (2012).

[16] D. Maryenko, J. Falson, Y. Kozuka, A. Tsukazaki, M. Onoda, H. Aoki, and M. Kawasaki, Phys. Rev. Lett. 108, 186803 (2012).

[17] J. Falson, D. Maryenko, Y. Kozuka, A. Tsukazaki, and M. Kawasaki, Appl. Phys. Express 4, 091101 (2011).

[18] K. F. Yang, H. W. Liu, T. D. Mishima, M. B. Santos, K. Nagase, and Y. Hirayama, New J. Phys. 13, 083010 (2011).
[19] B. Nedniyom, R. J. Nicholas, M. T. Emeny, L. Buckle, A. M. Gilbertson, P. D. Buckle, and T. Ashley, Phys. Rev. B 80, 125328 (2009).

[20] T. Gokmen, M. Padmanabhan, E. Tutuc, M. Shayegan, S. De Palo, S. Moroni, and G. Senatore, Phys. Rev. B 76, 233301 (2007).

[21] S. Das Sarma and E. H. Hwang, Phys. Rev. Lett. 84, 5596 (2000).

[22] E. Tutuc, S. Melinte, E. P. De Poortere, M. Shayegan, and R. Winkler, Phys. Rev. B 67, 241309 (2003).

[23] V. E. Kozlov, S. I. Gubarev, and I. V. Kukushkin, JETP Lett. 94, 397 (2011)

[24] F. Stern, Phys. Rev. Lett. 21, 1687 (1968).

[25] H. Tang and P. N. Butcher, J. Phys. C: Solid State Phys. 21, 3313 (1988).

[26] Y. Zhang and S. Das Sarma, Phys. Rev. Lett. 95, 256603 (2005).

[27] M. Padmanabhan, T. Gokmen, N. C. Bishop, and M. Shayegan, Phys. Rev. Lett. 101, 026402 (2008).

[28] T. Gokmen, M. Padmanabhan, K. Vakili, E. Tutuc, and M. Shayegan, Phys. Rev. B 79, 195311 (2009). 